\title{
DOUBLE NUMBERS IN GEOMETRY
}

\author{
NIHAL SIRIWARDENE \\ Department of Mathematics, University of Sri Jayewardenepura, Nugegoda, Sri Lanka.
}

(Date of receipt : 15 May 1987)

(Date of acceptance : 13 August 1987)

\begin{abstract}
The usual conplex numbers are often used to describe many familiar geometries. The Gauss interpretation as a 2 -dimensional sphere in 3 -dimensional Euclidean space and the Poincare interpretation as a double Lobachevski plane are some of them. Double numbers are also a type of complex numbers which differs the definition slightly. This algehra also yiclds many analogues applications to geometry. Pscudo-Euclidean geometry the basic framework of relativity, spherical and hyperbolic geometries are some of them.
\end{abstract}

\section{Introduction}

The relation of ordinary complex numbers to geometry is well known and have been dealt with extensively.

The double number is also a type of complex number which differs slightly in its definition; $\mathrm{i}^{2}=1$ insdead of -1 . This algebra was first introduced by the famous English geometer W. Clifford. The theory is quite classical. One may say it is as classical as ordinary complex numbers. Yet it is not familiar to many readers.

This article is an attempt to illuminate the connections of this algebra to geometry, especially to pseudo-Euclidean geometry, the basic framework of realtivity.

\section{Theorems}

An ordered pair of real numbers $\mathrm{a}$ and $\mathrm{b}$ is called a double number. Addition, multiplication are defined in an obvious way:

$$
\begin{aligned}
& (a, b)+(c, d)=(a+c, b+d) \\
& (a, b) \cdot(c, d)=(a c+b d, a d+b c)
\end{aligned}
$$

By the mapping $\mathrm{r} \longrightarrow(\mathrm{r}, 0)$ where $\mathrm{r}$ is real number, we can identify each double number of the form $(r, 0)$ with $r$. Hence if we denote the double number $(0,1)$ by $\mathrm{j}$, we then have 


$$
(a, b)=(a, 0)+(b, 0)(0,1)=a+b j
$$

and $(2.2)$ gives

$$
\mathbf{j}^{2}=1 \text {. }
$$

With these definitions the system of double numbers form a commutative algebra of dimension 2 over reals. We shall denote this algebra by $\mathbb{A}$.

If we write

$$
\mathrm{j}_{1}=1 / 2(1+\mathrm{j}) \text { and } \mathrm{j}_{2}=1 / 2(1-\mathrm{j})
$$

We find

$$
\mathrm{j}_{1}^{2}=\mathrm{j}_{1}, \mathrm{j}_{2}^{2}=\mathrm{j}_{2} \text { and } \mathrm{j}_{1} \mathrm{j}_{2}=0
$$

Hence by considering the basis $\mathrm{j}_{1}$ and $\mathrm{j}_{2}$ we see $\mathbb{A} \cong \mathbb{R} \times \mathbb{R}$

Where $\mathbb{R}$ denotes the set of real numbers. Each double number $Z=a+b j$ has a conjugate denoted by $\bar{z}$ which takes the form $a-b j$. It follows immediately

$$
\mathrm{z} \overline{\mathrm{Z}}=\mathrm{a}^{2}-\mathrm{b}^{2}
$$

For $\mathrm{z}_{1}, \mathrm{z}_{2} \in \mathbb{A} \quad$ we see

$\overline{\mathrm{z}_{1}+\mathrm{z}_{2}}=\overline{\mathrm{z}}_{1}+\overline{\mathrm{z}}_{2} \quad \overline{\mathrm{z}_{1} \mathrm{z}_{2}}=\overline{\mathrm{z}}_{1} \overline{\mathrm{z}}_{2}$

Real numbers

$$
|\mathrm{Z}|= \pm\left|\mathrm{a}^{2}-\mathrm{b}^{2}\right|^{1 / 2}
$$

are called modulii of $\mathrm{Z} \cdot(=\mathrm{a}+\mathrm{bj})$. Note that if

$$
\begin{gathered}
|\mathrm{Z}|=0 \text { then } \mathrm{a}= \pm \mathrm{b} \text { and vice versa. This implies } \\
\quad \mathrm{Z}=\mathrm{t}(1+\mathrm{j}) \text { or } \mathrm{Z}=\mathrm{t}(1-\mathrm{j})
\end{gathered}
$$

for some $t \in \mathbb{R}$

The double number with non-zero modulus can be expressed in polar form. For $r=|z|= \pm\left|a^{2}-b^{2}\right|^{1 / 2}$ we get

$$
\mathrm{Z}=\mathrm{a}+\mathrm{bj}=\mathrm{r}(\mathrm{a} / \mathrm{r}+\mathrm{b} / \mathrm{r} \mathbf{j})
$$


Since $(\mathrm{a} / \mathrm{r})^{2}-(\mathrm{b} / \mathrm{r})^{2}= \pm 1$, it follows

$\mathrm{a} / \mathrm{r}= \pm \operatorname{Cosh} \xi \quad \mathrm{b} / \mathrm{r}= \pm \operatorname{Sinh} \xi$

or

$$
\mathrm{a} / \mathrm{r}= \pm \operatorname{Sinh} \xi \quad \mathrm{b} / \mathrm{r}=+\operatorname{Cosh} \xi
$$

Where $\xi \in \mathbb{R}$ and $\cosh \xi$ and $\sinh \xi$ are the hyperbolic cosine and hyperbolic sine of the argument $\xi$

This gives

$Z=r(\cosh \xi+j \sinh \xi)$ or $Z=r(\operatorname{Sinh} \xi+j \cosh \xi), r \gtrless 0$

The argument of the double number $\mathrm{Z}$ is denoted by $\arg \mathrm{Z}$.

For $\mathrm{Z}_{1}, \mathrm{Z}_{2} \in \mathbb{A}$, it follows immediately

$$
\left|z_{1} z_{2}\right|=\left|z_{1}\right|\left|z_{2}\right|
$$

$\arg \mathrm{Z}_{1} \mathrm{Z}_{2}=\arg \mathrm{Z}_{1} \quad \arg \mathrm{Z}_{2}$ 
As with complex numbers, the double numbers can be represented as points of the plane. The following figure (a) describes the whole representation.

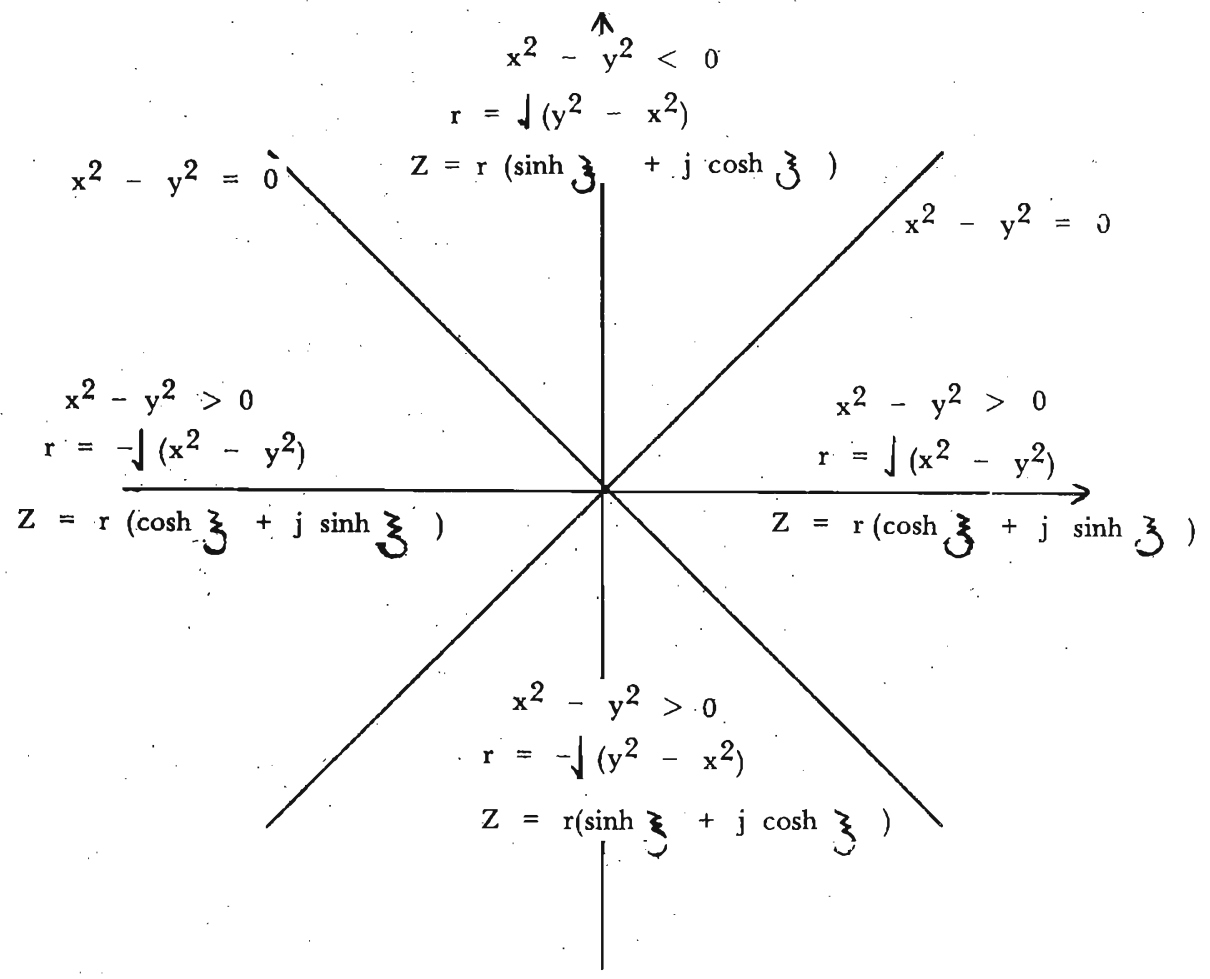

fig. (a) 
It is worth noting how $\mathbb{A}$ behaves as a ring. Other than $[0]$ and $\mathbb{A}$ the only ideals of $\mathbb{A}$ are

$$
\begin{aligned}
& I_{+}=\mathbb{R}(1+j)=\{r(1+j) / r \in \mathbb{R}\} \\
& I_{-}=\mathbb{R}(1-j)=\{r(1-j) / r \in \mathbb{R}\}
\end{aligned}
$$

It follows easily that

$$
\mathrm{I}_{+} \cap \quad \mathrm{I}_{-}=\{0\}
$$

and $\mathbb{A}-\left(I_{+} \cup I_{-}\right)$is the unit group $\cup$ of $\mathbb{A}$.

Now, what are the corresponding elements of $U$ in the plane'?

To see this let $\Phi=\{\operatorname{Cosh} \xi+j \operatorname{Sinh} \xi\}$

We see $P=\{r / r \in \mathbb{R}, \quad r>0\}$ and $P \Phi=\left\{r^{j} \mathcal{B} / r \in \mathbb{R}, r>0\right\}$ are subgroups of $U$. The subgroup $P \Phi$ fills the region I (see fig (a)).

- P $\Phi$ represents region III.Since

$$
j e^{j}=\operatorname{Sinh} \xi+j \operatorname{Cosh} \xi \text {, }
$$

we see $\mathrm{jP} \Phi$ and - jP $\Phi$ represents the regions II and IV respectively. Thus $U$ represents I $\cup$ II $U$ III $U$ IV. In general a point in the region is given by $u=r e j$. In region II it takes the form $u=$ rjej ${ }^{j}$.

The map $\quad Z \longrightarrow \bar{Z}$ is an automorphism of $\mathbb{A}$. As in complex numbers there are only two automorphisms namely the identity automorphism and the involutory automorphism.

The reader may verify whether the other properties in complex numbers do hold in this algebra. We shall now look at the geometries of this algebra.

If we consider as distance between $Z_{1}$ and $Z_{2}$ of the modulus of the difference $Z_{1}-Z_{2}$, we obtain the planar metric given by

$$
\mathrm{d}^{2}=\left|\mathrm{z}_{1}-\mathrm{z}_{2}\right|^{2}=\left|\mathrm{z}_{1}-\mathrm{z}_{2}\right|\left|\mathrm{z}_{1}-\mathrm{z}_{2}\right|
$$

For $\mathrm{Z}=\left(\mathrm{x}_{1}, \mathrm{y}_{1}\right)$ and $\mathrm{Z}=\left(\mathrm{x}_{2}, \mathrm{y}_{2}\right)$ we find

$$
\mathrm{d}^{2}=\left(\mathrm{x}_{1}-\mathrm{x}_{2}\right)^{2}-\left(\mathrm{y}_{1}-\mathrm{y}_{2}\right)^{2}
$$


It is evident from (5.2), the algebra with this metric is isomorphic to the 2 dimensional pseudo - Euclidean (Minowskian) plane ${ }^{1} \mathbb{R}_{2}$ of index 1 .

The Euclidean straight line

$$
\mathrm{bx}+\mathrm{cy}+\mathrm{d}=0
$$

with $b^{2}-c^{2} \neq 0$ is known as pseudo - Euclidean straight line, while (5.3) with $b^{2}-c^{2}=0$ is known as isotropic line in the pseudo - Euclidean plane. Recall in one-dimensional special relativity the line $b t+c y+d=0$ ( $\mathrm{t}$ is the time coordinate, $\mathrm{y}$ is the space coordinate) with $\mathrm{b}^{2}-\mathrm{c}^{2}>0$ is known as time - like line, and if $b^{2}-c^{2}<0$ it is known as space - like; the isotropic lines are known as light - like lines.

To each point $(x, y)$, if we associate the double number $Z=x+y j$, the above (5.3) takes the form

$$
(b+c j) Z+(b-c j) \vec{Z}+2 d=0
$$

With $(b+c j)(b-c j) \neq 0$. Thus every pseudo - Euclidean straight line can be represented by an equation of the form:

$$
\alpha Z_{1}+\bar{\alpha} \bar{Z}+\gamma=0
$$

With $\alpha \bar{\alpha} \neq 0$, The isotropic line also take the form (5.5) with $\alpha \bar{\alpha}=0$.

The equation of the pseudo - Euclidean circle could be worked out similarly. Observe that, this pseudo-Euclidean circle is a hyperbola. The geometry of pseudo Euclidean plane may be worked out in an analogus manner.

The ordered pair $\left(Z_{1}, Z_{2}\right)$ is said to be an element of the projective line $P(\mathbb{A})$ over $\mathbb{A}$ whenever not both elements $Z_{1}, Z_{2}$ lie in the same ideal I $(\neq \mathbb{A})$ of $\mathbb{A}$.

The two ordered pairs $\left(Z_{1}, Z_{2}\right)$ and $\left(Z_{1}^{\prime}, Z_{2}^{\prime}\right)$ represent the same element whenever $Z_{i}^{\prime}=r Z_{i}(i=1,2)$ for some $r \in \cup$ (see 4.1). This enables to identify the pseudo - Euclidean points with $\mathrm{P}(\mathbb{A})$. The point $(\mathrm{x}, \mathrm{y})$ can be identified with $(\mathrm{x}+\mathrm{yj}, 1) \mathrm{P}(\mathbb{A})$. The pseudo - Euclidean point $\infty$ identified with $(1,0) \in \mathrm{P}(\mathbb{A})$. The isotropic lines are identified with $\left(Z_{1}, 1+j\right)$ and $\left(Z_{1}, 1-j\right)$ of $P(\mathbb{A})$, where $Z_{1} \notin I_{+}$and $Z_{1}^{\prime} \notin I_{-}$.

This identification enables one to describe the geometry of $\mathrm{P}(\mathbb{A})$. The interested reader may refer (1). 
The elements of $\mathbb{A}$ which do not have inverses are the elements of the ideals $I_{+}$and I_of $\mathbb{A}$

If we supplement the algebra by these ideal elements then it is possible to introduce into this algebra spherical and hyperbolic metrics:

$$
\begin{aligned}
& \operatorname{Cos}^{2} \frac{d}{2 r}=\frac{\left|1+z_{1} \bar{z}_{2}\right|^{2}}{\left(1+\left|z_{1}\right|^{2}\right) 2\left(1+\left|z_{2}\right|^{2}\right)} \\
& \operatorname{Cosh}^{2} \frac{d}{2 r}=\frac{\left|1-z_{1} \bar{z}_{2}\right|^{2}}{\left(1-\left|z_{1}\right|^{2}\right)\left(1-\left|z_{2}\right|^{2}\right)}
\end{aligned}
$$

Where $d$ is the distance between the points $Z_{1}$ and $Z_{2}$ and $r$ is the radius $S_{2}$ the 2 - dimensional sphere in pseudo - Euclidean space ${ }^{1} \mathbb{R}_{3}$ of index 1 .

As in ordinary complex numbers, constructing a stereographic projection, it is possible to represent the supplemented algebra as the unit sphere.

$$
S=\left\{\left(x_{1}, x_{2}, x_{3}\right) / x_{1}^{2}+x_{2}^{2}-x_{3}^{2}=1\right\}
$$

in ${ }^{1} \mathbb{R}_{3}$. It is easier to verify that this supplemented algebra with spherical metric is isometric to the 2 - dimensional sphere of radius $r$ in ${ }^{1} \mathbb{R}_{3}$. In the case of hyperbolic metric it is isometric to 2 -dimensional sphere of imaginary radius in the space $\mathbb{R}_{3}$ of index 2 . The algebra $\mathbb{A}$ with the speherical \& hyperbolic metrics was first studied in (4). and (5).

It has been shown in (2) that the pseudo - Euclidean plane supplemented by the ideal elements with the group of motions.

$$
\mathrm{Z}^{\prime}=\frac{\alpha \mathrm{Z}+\beta}{\gamma \mathrm{Z}+\delta}
$$

Where the elements of the matrix $\left(\begin{array}{ll}\alpha & \beta \\ \gamma & \delta\end{array}\right) \quad$ satisfy $\alpha \bar{\alpha}+\beta \bar{\beta}=1$ $\gamma \gamma+\delta \bar{\delta}=1, \quad \alpha \bar{\gamma}+\overline{\beta \delta}=0$

in spherical metric and $\alpha \bar{\alpha}-\beta \bar{\beta}=1, \gamma \bar{\gamma}-\delta \bar{\delta}=1$; $\alpha \bar{\gamma}-\beta \bar{\delta}=0$ in hyperbolic metric, represent models of geometries of constant curvature. These models are to a large extent similar to the known models of Poincare for non-Euclidean geometries. They are known as tangential models of Poincare. 
7. It is natural to generalize the above geometries to $n$-dimensions.

The affine space $A_{n}(\mathbb{A})$ over $\mathbb{A}$ is introduced as follows. The points of $A_{n}(\mathbb{A})$ are characterised as system of $n-$ arguments.

$$
\mathrm{z}_{1}, \mathrm{z}_{2}, \ldots \ldots \ldots \ldots, \mathrm{z}_{\mathrm{n}}
$$

of $\mathbb{A}$. The affine space over $\mathbb{A}$, with metric

$\mathrm{d}^{2}=\Sigma\left|\mathrm{z}_{\mathrm{i}}-\mathrm{z}_{\mathrm{i}}^{\prime}\right|^{2}=\sum\left(\mathrm{z}_{\mathrm{i}}-\mathrm{z}_{\mathrm{i}}^{\prime}\right)\left(\overline{\mathrm{z}}_{\mathrm{i}}-\overline{\mathrm{z}}_{\mathrm{i}}^{\prime}\right)$

is known as unitary - Euclidean space $\cup_{\mathbf{n}}(\mathbb{A})$.

It is easy to verify, the space $U_{n}(\mathbb{A})$ is isometric to pseudo Euclidean space ${ }^{n} \mathbb{R}_{2 \mathrm{n}}$ of index $\mathrm{n}$.

The projective space $P_{n}(\mathbb{A})$ over $\mathbb{A}$ is introduced as follows. The points of $P_{n}(\mathbb{A})$ are characterised as system of $n$-arguments.

$$
\alpha \mathrm{Z}_{1}, \alpha \mathrm{Z}_{2}, \ldots \ldots \ldots \ldots \ldots \ldots, \alpha \mathrm{Z}_{\mathrm{n}+1}
$$

where $\alpha$ is an arbitrary element of the algebra.

The projective space $\mathrm{P}_{\mathrm{n}}(\mathbb{A})$ with metric

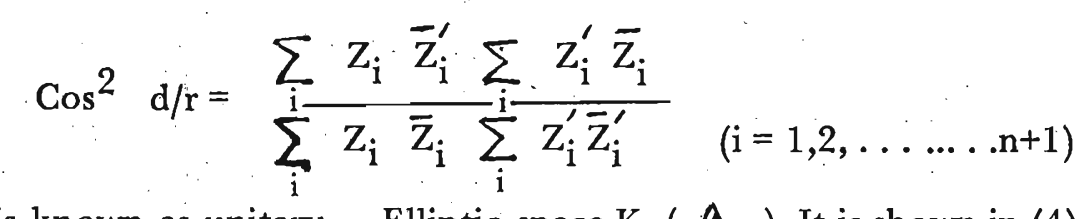

is known as unitary - Elliptic space $K_{n}(\mathbb{A})$. It is shown in (4), The space $\mathrm{K}_{\mathrm{n}}(\mathbb{A})$ is isometric to pseudo - Riemann space ${ }^{\mathrm{n}} \mathrm{V}_{2 \mathrm{n}}$ of index $\mathrm{n}$. For further study of these spaces, I refer the reader to (2).

\section{References}

1. BENZ, W. (1973) VorlesungenUber Geometrie der Alegebren Springer Verlag.

2. DUBNOW, Ya. S. (1933) On tensors with non-scalar components, Trudi Seminara po Vectornomui tenzornomu analizu, pri MGU, 1 196-222.

3. ROZENFEL'D, B.A. \& YAGLOM, I.M. (1951) Mathematicheskii Sbornik 28(1).

4. YAGLOM; I.M. (1949) Projective metric definitions in the plane and Complex numbers. Trudi Seminara po Vektornomui tenzornomu analizu pri MGU, 7: $276-300$. plane geometrics with constant Curvature. DAN SSSR, V.L III 405-408. 